\title{
Duly noted: Lessons from a two-site intervention to assess and improve the quality of clinical documentation in the electronic health record
}

Laura Fanucchi'; Donglin Yan²; Rosemarie L. Conigliaro ${ }^{3}$

${ }^{1}$ Center for Health Services Research, University of Kentucky College of Medicine, Lexington, KY;

${ }^{2}$ University of Kentucky College of Public Health, Lexington, KY;

${ }^{3}$ Albert Einstein College of Medicine/Montefiore Medical Center, Bronx, NY

\section{Keywords}

Electronic health record, clinical documentation, medical education

\section{Summary}

Background: Communication errors are identified as a root cause contributing to a majority of sentinel events. The clinical note is a cornerstone of physician communication, yet there are few published interventions on teaching note writing in the electronic health record (EHR). This is a prospective, two-site, quality improvement project to assess and improve the quality of clinical documentation in the EHR using a validated assessment tool.

Methods: Internal Medicine (IM) residents at the University of Kentucky College of Medicine (UK) and Montefiore Medical Center/Albert Einstein College of Medicine (MMC) received one of two interventions during an inpatient ward month: either a lecture, or a lecture and individual feedback on progress notes. A third group of residents in each program served as control. Notes were evaluated with the Physician Documentation Quality Instrument 9 (PDQI-9).

Results: Due to a significant difference in baseline PDQI-9 scores at MMC, the sites were not combined. Of 75 residents at the UK site, 22 were eligible, 20 (91\%) enrolled, 76 notes in total were scored. Of 156 residents at MMC, 22 were eligible, 18 (82\%) enrolled, 40 notes in total were scored. Note quality did not improve as measured by the PDQI-9.

Conclusion: This educational quality improvement project did not improve the quality of clinical documentation as measured by the PDQI-9. This project underscores the difficulty in improving note quality. Further efforts should explore more effective educational tools to improve the quality of clinical documentation in the EHR.

Correspondence to:

Laura Fanucchi, MD, MPH

Center for Health Services Research

Assistant Professor of Medicine

University of Kentucky College of Medicine

900 South Limestone

306B Charles T. Wethington Bldg

Lexington, KY 40536

Ph: 859-323-1982

Fax: 859-257-2605

Email: laura.fanucchi@uky.edu
Appl Clin Inform 2016; 7: 653-659

http://dx.doi.org/10.4338/ACl-2016-02-CR-0025

received: February 29, 2016

accepted: May 9, 2016

published: July 6, 2016

Citation: Fanucchi L, Yan D, Conigliaro RL. Duly noted: Lessons from a two-site intervention to assess and improve the quality of clinical documentation in the electronic health record. Appl Clin Inform 2016; 7: 653-659 http://dx.doi.org/10.4338/ACI-2016-02-CR-0025 


\section{Background}

Electronic health records (EHRs) have largely ameliorated the access and legibility limitations of paper records. Conversely, the widespread use of EHR efficiency tools like copy-forward infringes on the validity of notes, and contributes to communication errors [1-3]. Communication errors are identified as a root cause contributing to a majority of sentinel events [4].

The clinical note is a cornerstone of communication, yet there are few published interventions on improving note writing in the EHR [5-7]. Two tools validated for evaluating note quality, the Physician Documentation Quality Instrument 9 (PDQI-9) for inpatient notes [8], and QNOTE for outpatient notes [9], have not been applied in an educational intervention to improve the quality of notes.

Our study is a prospective quality improvement (QI) project in the internal medicine (IM) residency training programs at the University of Kentucky College of Medicine (UK) in Lexington, KY, and Montefiore Medical Center/Albert Einstein College of Medicine (MMC) in Bronx, NY. The aim of the project is to assess and improve clinical documentation quality using the PDQI- 9 as a scoring and feedback tool. The study was approved by the Institutional Review Board (IRB) of both institutions.

\section{Methods}

The QI project took place between July and September, 2013. At UK, IM resident physicians (PGY1-3) were identified via review of the clinical rotation schedule, divided into three project groups, and informed consent was obtained. At MMC, IM residents are divided into three Firms, which was the basis for the project groups (informed consent not required). The three groups were: lecture plus feedback (LF), lecture (L), and control (C). LF and L groups included residents who attended an hour-long lecture and were on an inpatient medicine rotation during the project period, whereas the $\mathrm{C}$ group was on elective or other non-ward rotation during this same time. At UK, residents who were unable to attend the lecture (due to night shifts or vacation) were assigned to the $\mathrm{C}$ group by default and served as controls. At both sites, progress notes written during the month prior to the lecture were scored for the L and LF groups using the PDQI-9 and compared to a second set of progress notes written after the lecture took place. The LF group received an additional individual feedback session at which they reviewed the results of the PDQI-9 from their pre-lecture set of notes with a faculty member. A "set" of progress notes was defined as two consecutive notes written by the same resident about the same patient; the later note was scored with the PDQI-9 and the earlier note served as reference to assess copy-paste and originality. Progress notes were identified within the EMR by searching by resident name and date range and selecting patients from the list obtained using a random number generator. Charts with fewer than two consecutive days of progress notes were excluded.

The project leaders ran the hour-long didactic lecture sessions, which consisted of a PowerPoint presentation describing the elements of a good note, with examples of appropriate and inadequate documentation. Multiple blinded faculty at each site scored notes and provided feedback. Feedback sessions were one-on-one with the resident and faculty member reviewing notes and PDQI-9 results.

The summary PDQI-9 scores for the three groups were treated as ordinal variables and the median pre-post score differences of each group were compared using the Kruskal-Wallis Test. All statistical analyses were conducted using NPAR1WAY procedure in SAS ${ }^{\curvearrowleft}$ version 9.3.

\section{Results}

\section{Participation rate}

At UK, 22 out of a total of 75 IM residents were on inpatient wards rotations during the 3-month project period, and 20 enrolled for a $91 \%$ participation rate. There were 9 residents in the LF group, 
5 in the L, and 6 in the $C$ groups. One resident in the LF group was excluded because the feedback session did not occur. Two sets of notes for each resident were evaluated with the PDQI-9, for a total of 76 notes ( $\triangleright$ Figure 1 ).

At MMC, 22 out of a total of 156 IM residents were on inpatient wards rotations, and 18 enrolled for an $82 \%$ participation rate. There were 5 residents in the LF group, 8 residents in the L group, and 5 in the $\mathrm{C}$ group. For the 8 residents in the L group the lecture session occurred earlier than planned, leaving no pre-intervention notes; thus, these 8 residents were excluded from analysis. For the LF and $\mathrm{C}$ groups, a total of 40 notes were evaluated ( $>$ Figure 1 ).

\section{Note Evaluation}

The PDQI-9 scores for the three groups at UK are summarized in $>$ Table 1 . There were no statistically significant differences in baseline PDQI-9 scores between the 3 groups $\chi^{2}(2, N=19)=1.92, p=$ .38 , nor did the scores change significantly pre- and post- intervention $\chi^{2}(2, \mathrm{~N}=19)=4.67, \mathrm{p}=.10$ $(>$ Table 1).

At MMC, there was a statistically significant difference in the baseline PDQI-9 scores between LF and $C$ groups $\chi^{2}(1, N=10)=8.11, p=0.004$. No further comparisons were made, and the data for the two sites were not combined.

\section{Lessons Learned}

We anticipated that individual feedback would be more effective than a group lecture in changing resident behavior around clinical documentation, and that both would be better than no educational intervention. However, our intervention to assess and improve the quality of clinical documentation was not successful as measured by the PDQI-9. While we hoped that conducting the same project at two training programs would improve our ability to detect a change, we were unable to combine the data because of the significant differences in baseline PDQI-9 scores. As the PDQI-9 had been previously validated, and did not include recommendations for evaluator training, we did not conduct training or benchmarking sessions. On further review of the MMC data, we found that one rater consistently scored notes much higher than other raters (data not shown). This observation suggests that it is important to calibrate raters when using the PDQI-9.

There are other important limitations to our educational intervention. It occurred over a short period of time, and the time period for notes obtained pre-didactic was not consistent between the LF and L groups due to variability in resident schedules. The sample size was small, thus we were unable to adjust for resident seniority. Furthermore, though MMC has a note template, we did not create one at UK, nor did we focus the didactic lecture or feedback on a template or specific format.

Consideration of the properties of complex adaptive systems, such as nonlinearity, emergence, coevolution, and patterns of interaction [10] is highly relevant to efforts to improve the quality of clinical documentation. Melvin, et al (2015) found that feedback coupled with a written communication tutorial improved the quality of medical student documentation as measured by their internally-developed tool [5]. These authors included a curriculum on giving feedback, which speaks to the emergence and coevolution aspects of complex adaptive systems, and may have made their intervention more successful. We did not assess the skills of our faculty to give feedback on quality note documentation, nor did we provide any training in this skill. In addition, we did not specify the use of a standardized template, which may improve some elements of note quality and make evaluation less subjective simply by encouraging a pattern of interaction and standard work [6, 7]. Thus our faculty may have been hindered in rendering an objective assessment of note quality, despite using a validated tool.

Watling, et al (2012) have suggested that residents are most engaged when learning objectives are clearly and immediately imbedded in their clinical work [11], an illustration of the emergence aspect of complexity theory. Feedback efforts, therefore, while highly emphasized in medical education, may fail if they lack such contextual credibility. For example, March, et al (2016) showed a wide variability in intern's clinical documentation even when using a simulated exercise that closely approximated clinical care [12]. In keeping with the behaviors of complex adaptive systems, perhaps 
our intervention fell short due to the residents' relatively low perceived importance associated with clinical documentation, the many competing demands on trainee time, and minimal incentive to create documentation that is accurate and of high quality.

\section{Conclusion}

In conclusion, our educational quality improvement project did not improve the quality of clinical documentation for a group of IM trainees as measured by the PDQI-9. This project underscores the difficulty in improving note quality despite validated instruments and individualized feedback. Our intervention may not have been successful due to its short duration as well as the characteristics of complex adaptive systems that we were unable to address. Residency is the time when clinical behaviors essential to quality care are solidified [13], and it is a time of tremendous opportunity for teaching best practices for communicating through the EHR. We suggest that prepping educators in the available assessment tools is likely important, and we anticipate that ongoing longitudinal interventions will be more effective than time-limited interventions.

\section{Conflict of Interest Statement}

The authors declare no commercial associations that may pose a conflict of interest with the submitted article.

\section{Protection of Human Subjects}

The study was performed in compliance with the World Medical Association Declaration of Helsinki on Ethical Principles for Medical Research Involving Human Subjects, and was reviewed by the Institutional Review Boards of the University of Kentucky College of Medicine and Montefiore Medical Center/Albert Einstein College of Medicine (MMC)

\section{Author Contribution Statement}

Laura Fanucchi and Rosemarie Conigliaro conceived of and designed the study, acquired data, and drafted the manuscript. Donglin Yan contributed to data analysis and interpretation and drafted sections of the manuscript. All listed authors approved the final version of the manuscript.

\section{Funding}

The authors report no external funding source for this study. 


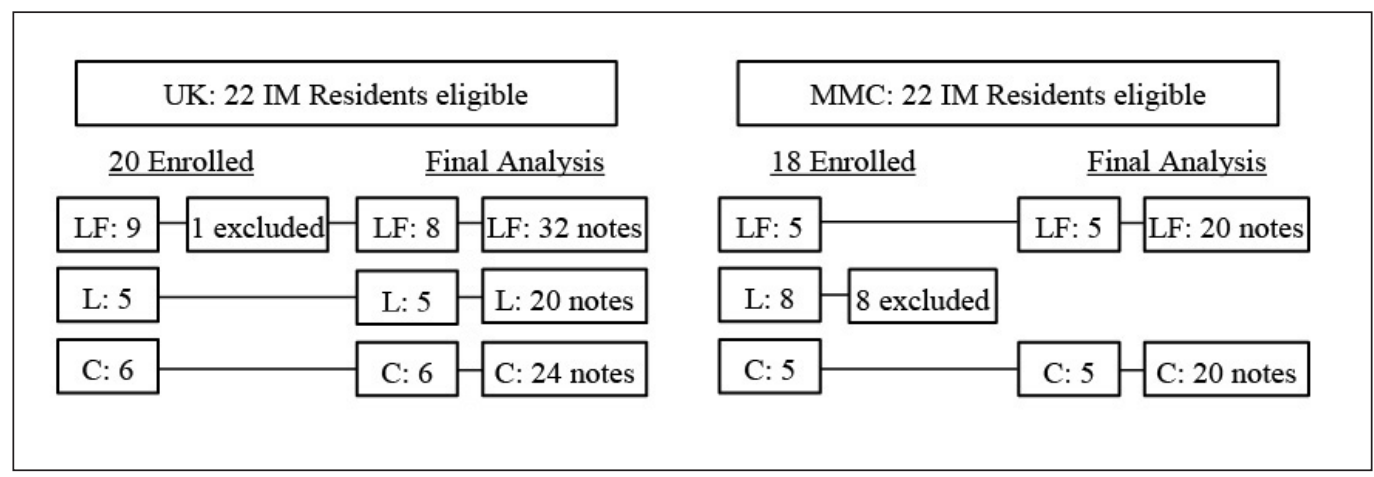

Fig. 1 Project enrollment flow diagram; LF: lecture plus feedback, L: lecture only, C: no intervention control group. 
Table 1 PDQI-9 scores pre- and post-educational intervention for the three groups at UK.

\begin{tabular}{|l|l|l|l|l|}
\hline Group (n) & $\begin{array}{l}\text { Lecture plus } \\
\text { Feedback (8) }\end{array}$ & Lecture Only (5) & Comparison (6) & $\boldsymbol{p}$ \\
\hline PDQI-9 Pre-score, Median (range) & $35(31-42)$ & $31(29-37)$ & $35(22-38)$ & 0.38 \\
\hline PDQI-9 Post-score, Median (range) & $32.5(30-42)$ & $39(24-45)$ & $35.5(28-39)$ & \\
\hline PDQI-9 Difference, Median (range) & $-1.5(-7-7)$ & $8(-6-9)$ & $1.5(-1-9)$ & 0.10 \\
\hline
\end{tabular}




\section{References}

1. Thornton JD, Schold JD, Venkateshaiah L, Lander B. Prevalence of copied information by attendings and residents in critical care progress notes. Critical Care Medicine 2013; 41(2): 382-388.

2. Heiman HL, Rasminsky S, Bierman JA, Evans, DB, Kinner, KG, Stamos, J, Martinovich, Z, McGaghie, WC. Medical students' observations, practices, and attitudes regarding electronic health record documentation. Teaching and Learning in Medicine 2014; 26(1): 49-55.

3. Weis JM, Levy PC. Copy, paste, and cloned notes in electronic health records: Prevalence, benefits, risks, and best practice recommendations. Chest 2014; 145(3): 632-638.

4. Sentinel Event Data - Root Causes by Event Type. The Joint Commission; 2015. http://www.jointcommis sion.org/assets/1/18/Root_Causes_Event_Type_2004-2Q_2015.pdf. Accessed October 5, 2015.

5. Melvin L, Connolly K, Pitre L, Dore KL, Wasi P. Improving medical students' written communication skills: design and evaluation of an educational curriculum. Postgraduate Medical Journal 2015; 91(1076): 303-308.

6. Dean SM, Eickhoff JC, Bakel LA. The effectiveness of a bundled intervention to improve resident progress notes in an electronic health record. Journal of Hospital Medicine 2015; 10(2): 104-107.

7. Grogan EL, Speroff T, Deppen SA, Roumie CL, Elasy TA, Dittus RS, Rosenbloom ST, Holzman MD. Improving documentation of patient acuity level using a progress note template. Journal of the American College of Surgeons 2004; 199(3): 468-475.

8. Stetson PD, Bakken S, Wrenn JO, Siegler EL. Assessing Electronic Note Quality Using the Physician Documentation Quality Instrument (PDQI-9). Applied Clinical Informatics 2012; 3(2): 164-174.

9. Burke HB, Hoang A, Becher D, Fontelo P, Liu F, Stephens M, Pangaro LN, Sessums LL, O’Malley P, Baxi NS, Bunt CW, Capaldi VF, Chen JM, Cooper BA, Djuric DA, Hodge JA, Kane S, Magee C, Makary ZR, Mallory RM, Miller T, Saperstein A, Servey J, Gimbel RW. QNOTE: an instrument for measuring the quality of EHR clinical notes. Journal of the American Medical Informatics Association: JAMIA: the Journal of the American Medical Association 2014; 21(5): 910-916.

10.Sturmberg JP, Martin CM, Katerndahl, DA. Systems and complexity thinking in general practice literature: An integrative, historical narrative review. Annals of Family Medicine 2014; 12(1): 66-74.

11. Watling C, Driessen E, van der Vleuten CPM, Lingard L. Learning from clinical work: the roles of learning cues and credibility judgements. Medical Education 2012; 46: 192-200.

12. March CA, Scholl G, Dversdal RK, Richards M, Wilson LM, Mohan V, Gold JA. Use of Electronic Health Record Simulation to Understand the Accuracy of Intern Progress Notes. Journal of Graduate Medical Education 2016; 8(2): 237-240.

13. Asch DA, Nicholson S, Srinivas S, Herrin J, Epstein AJ. Evaluating obstetrical residency programs using patient outcomes. JAMA: the Journal of the American Medical Association 2009; 302(12): 1277-1283. 